\title{
Deactivating Cochlear Implant Electrodes Based on Pitch Information for Users of the ACE Strategy
}

\author{
Deborah Vickers, Aneeka Degun, Angela Canas, Thomas Stainsby \\ and Filiep Vanpoucke
}

\begin{abstract}
There is a wide range in performance for cochlear implant (CI) users and there is some evidence to suggest that implant fitting can be modified to improve performance if electrodes that do not provide distinct pitch information are de-activated. However, improvements in performance may not be the same for users of all CI devices; in particular for those with Cochlear devices using n-of-m strategies (ACE or SPEAK).

The goal of this research was to determine for users of Cochlear devices (CP810 or CP900 series processors) if speech perception could be improved when indiscriminable electrodes were de-activated and this was also compared to when the same number of discriminable electrodes were de-activated.

A cross-over study was conducted with 13 adult CI users who received experimental maps with de-activated channels for a minimum of 2 months and these were compared to optimised clinical maps.

The findings showed that there were no significant benefits of electrode deactivation on speech perception and that there was a significant deterioration in spectro-temporal ripple perception when electrodes were switched off. There were no significant differences between de-activation of discriminable or indiscriminable electrodes.

These findings suggest that electrode de-activation with n-of-m strategies may not be beneficial.
\end{abstract}

D. Vickers $(\bowtie) \cdot$ A. Degun $\cdot$ A. Canas

UCL Ear Institute, 332 Grays Inn Road, London WC1X 8EE, UK

e-mail: d.vickers@ucl.ac.uk

A. Degun

e-mail: aneeka.degun.10@ucl.ac.uk

A. Canas

e-mail: a.canas@ucl.ac.uk

T. Stainsby $\cdot$ F. Vanpoucke

Cochlear Technology Centre, Schaliënhoevedreef 20, building I, 2800 Mechelen, Belgium

(C) The Author(s) 2016

P. van Dijk et al. (eds.), Physiology, Psychoacoustics and Cognition in Normal

and Impaired Hearing, Advances in Experimental Medicine and Biology 894,

DOI 10.1007/978-3-319-25474-6 13 
Keywords Cochlear implant - Electrode discrimination - Electrode deactivation Fitting $\cdot$ Mapping $\cdot$ Electrode-neurone interface $\cdot$ Speech perception $\cdot$ Pitch ranking $\cdot$ ACE strategy $\cdot$ n-of-m

\section{Introduction}

Various approaches have been explored to look at the effects of re-mapping cochlear implants (CIs) to overcome the problems associated with neural dead regions or poor electrode placement (electrode-neuron interface (ENI)). The mapping interventions have typically explored ways to identify electrodes for de-activation using Computerized Tomography (CT), threshold profiling with focussed stimulation, detection of modulation and pitch-based electrode discrimination. Electrodes indicated as being in a region with a poor ENI have typically been deactivated with the intention of improving spectral resolution by reducing overlapping electrode stimulation patterns.

If a poor ENI arises due to an underlying neural dead region, the transmission of information in that frequency channel could be impeded; if a poor ENI arises due to the presence of broad electrical fields, this will lead to corruption of channel specific information. For both cases it could be beneficial to deactivate electrodes, however when doing so frequency information is re-allocated to other electrodes making analysis bands broader and distorting frequency-to-electrode mapping. These effects are small for individual electrode deactivation but can be substantial for deactivation of multiple electrodes, potentially resulting in poorer precision of information transmission; a completely opposite effect to that intended.

Some n-of-m (n channels with highest input amplitudes stimulated out of a possible m channels; e.g. ACE (8-of-22) or SPEAK) strategies may not benefit from deactivation of electrodes due to the simplification of the information delivery already inherent in the strategy.

The findings from electrode deactivation studies are mixed. Noble et al. (2014) used a CT-guided technique in which pre- and post-operative scans were used to determine the electrode positioning with respect to the modiolus. Poorly positioned electrodes were deactivated and additional mapping adjustments made. Assessments were conducted at baseline and following a 3-6 week exposure period with the adjusted map. Learning effects were not accounted for. Thirty-six out of 72 (50\%) participants demonstrated a benefit on one outcome measure (CNC words, AzBio sentences (quiet, $+10 \mathrm{~dB},+5 \mathrm{~dB}$ signal-to-noise ratio (SNR)) and BKBSin), 17 had a significant degradation in performance $(24 \%)$, three cochlear users had mixed results $(4 \%)$ and the final 16 participants did not demonstrate any effect of the de-activation (22\%). When broken down by device, the results showed significant benefit on one measure for 16 out of 26 AB and MEDEL users $(62 \%$; seven showed significant deterioration) and 20 out of 46 Cochlear users ( $44 \%$; ten showed significant deterioration and three a mixture of significant deterioration and significant improvement). Overall the results demonstrated significant improvements in 
speech understanding in quiet and noise for re-mapped participants, with no particular test exhibiting the greatest improvements. Cochlear users were less likely to benefit from electrode deactivation than users of the other devices. As speculated earlier it could be that the n-of-m strategies used in the Cochlear device may be less sensitive to channel overlap effects because not all channels are stimulated in each cycle.

Zwolan et al. (1997) assessed users of an older generation Cochlear device (Mini22) stimulated in bipolar mode using an MPEAK sound processing strategy. They demonstrated positive results when deactivating electrodes based on pitch discrimination. Testing was acute with care to randomise between clinical and experimental maps in the assessment, but the experimental map exposure was far briefer than that of the clinical map. They found significant improvements in speech perception in at least one speech test for seven out of nine participants; while two participants showed a significant decrease on at least one speech test. This study demonstrated some benefit from channel deactivation for Cochlear users; however the MPEAK strategy was based on feature extraction and not n-of-m.

Work by Saleh (Saleh et al. 2013; Saleh 2013) using listeners with more up-todate sound processing strategies also demonstrated positive findings when deactivating electrodes. They based channel selection on acoustic pitch ranking. Electrodes were identified for deactivation if they did not provide distinct or tonotopic pitch information. Twenty-five participants were enrolled. They received takehome experimental maps for a 1-month time period before being assessed. Assessments were conducted with the clinical map at baseline and again following experience with the experimental map. They found statistically significant improvements for electrode deactivation on speech perception tests for 16 of the 25 participants (67\%). For the Cochlear users 56\% (five out of nine) gained benefit compared to $69 \%$ for the AB and MED-EL users (11 out of 16). Participant numbers were small but there was a trend for fewer Cochlear users to benefit compared to other devices; similar to that observed in Noble et al. (2014).

Garadat et al. (2013) deactivated electrodes based on modulation detection in a group of ACE users. Up to five electrodes were switched off and they were selected such that only individual non-adjacent contacts were deactivated to avoid large regions being switched off. Following deactivation they demonstrated significant benefit for consonant perception and CUNY sentences in speech-shaped noise ( 8 out of 12 showed benefit) but demonstrated a deterioration in performance for vowel perception (7 out of 12 showed degradation). Testing was acute so learning effects were not controlled for and listeners were not given time to adapt to the experimental maps; with further experience and acclimatisation users may have performed better on the vowel task, particularly because it is known that vowel perception is sensitive to filtering adjustments (Friesen et al. 1999). These findings are potentially positive for ACE users but without a control condition they are not definitive proof.

Some researchers have not observed benefit from electrode deactivation and have even demonstrated potentially deleterious effects for Cochlear device users. Henshall and McKay (2001) used a multidimensional scaling technique to identify 
and deactivate electrodes having non-tonotopic percepts. They did not find an improvement in speech perception when channels were switched off and hypothesized that this could be due to the shift in frequency-to-electrode allocation produced by clinical software when electrodes are deactivated; particularly in the apical region.

The findings from different deactivation studies are inconclusive, partly due to the difficulties of comparing experimental to clinical maps that the CI users had adapted to and partly due to the impact that deactivating channels can have on other factors (e.g. rate of stimulation; filter-frequency allocation) which differ between devices and sound processing strategies. There is a trend suggesting that deactivating electrodes may be less effective for ACE or SPEAK users than for the CIS based sound processing strategies incorporated in $\mathrm{AB}$ or MEDEL devices.

The goal of this research was to explore the impact of electrode deactivation for adult users of the ACE strategy ensuring that participants received sufficient adaptation time with experimental maps and that learning effects were controlled for. Additionally participants' clinical maps were optimised to ensure comparisons were made with an optimal control condition. A pitch ranking task was used to identify indiscriminable electrodes for deactivation and an additional control condition was included in which discriminable electrodes were deactivated to determine if any benefits observed were due to switching off electrodes with a poor ENI or just due to having fewer channels.

The research objectives were:

1. To determine if deactivating electrodes that are indiscriminable based on pitch ranking leads to significant changes in speech perception for adult ACE users when compared to an optimised clinical map.

2. To determine if deactivating the same number of discriminable electrodes leads to significantly different performance to the deactivation of indiscriminable electrodes.

\section{Method}

\subsection{Ethics Approval}

Research was ethically approved by National Research Evaluation Service (PRSC 23/07/2014).

\subsection{Participants}

Thirteen post-lingually deafened adult ACE users with Cochlear CP810 or CP900 series devices were recruited. Individuals with ossification or fibrosis were excluded and participants had to have a minimum of 12 months CI experience and 
primarily speak English. Median age was 67 years (range 36-85 years); the median length of CI use was 2 years (mean 4 years). Median duration of severe-to-profound deafness prior to surgery was 4 years (mean 2 years).

\subsection{Study Design}

A crossover single-blinded (participant unaware of mapping interventions) randomised control trial was conducted. Prior to starting each participant had their clinical map optimised by adjusting individual channel levels and they received a minimum of 1 month acclimatisation to this optimised map. The crossover study used an A-B-B-A/B-A-A-B design in which map A had indiscriminable electrodes deactivated and map $\mathrm{B}$ had an equal amount of discriminable electrodes deactivated.

\subsection{Equipment}

For the majority of the assessments the sound processor was placed inside an Otocube; a sound proof box designed for testing CI users to simulate listening over a loudspeaker in a sound treated room. Stimulus delivery and collection of responses was controlled using a line in from a laptop to the Otocube and sounds were presented over the Otocube loudspeaker positioned at $0^{\circ}$ azimuth to the sound processer.

The direct stimulation pitch-ranking task was conducted using the Nucleus Implant Communicator (NIC) to send stimuli directly to individual electrodes.

\subsection{Test Materials}

\section{CHEAR Auditory Perception Test (CAPT)}

The CAPT was used because it is sensitive to spectral differences in hearing aid fitting algorithms (Marriage et al. 2011) and was known to be highly repeatable (inter-class correlation of 0.70 for test and re-test conditions; Vickers et al. 2013).

The CAPT is a four-alternative-forced-choice monosyllabic word-discrimination test spoken by a female British English speaker. It contained ten sets of four minimally-contrastive real words; eight sets with a contrastive consonant, e.g. fat, bat, cat, mat and two sets with a contrastive vowel, e.g. cat, cot, cut, cart. The participant selected from four pictures on a computer screen. The d' score was calculated.

Stimuli were presented at $50 \mathrm{dBA}$ in quiet; a level selected in pilot work because it resulted in performance falling on the slope of the psychometric function.

\section{Children's Coordinate Response Measure (CCRM)}

The adaptive CCRM was based on the test developed by Bolia et al. (2000) and Brungart (2001). It was used because of low contextual cues, and ease of task. 
Each stimulus sentence took the form:

'Show the dog where the (colour) (number) is?': e.g. 'show the dog where the green three is?'

There were six colour options (blue, black, green, pink, red and white) and eight possible numbers (1-9, excluding 7). Stimuli were spoken by a British female speaker.

Two different maskers were used: 20-talker babble (created by modulating a speech-shaped noise with the amplitude envelope of male sentences) or speechshaped noise (average long-term spectrum of the sentences). Random sections of the noise were selected on each trial.

The sentences were presented at $65 \mathrm{dBA}$ and the noise adjusted adaptively (2-down/1-up) on the basis of whether or not both the colour and number were identified correctly. Initial step size was $9 \mathrm{~dB}$, and decreased after two reversals to $3 \mathrm{~dB}$. A further four reversals were run and averaged to obtain the speech reception threshold (SRT), but no test was longer than 26 trials.

\section{Spectral-Temporally Modulated Ripple Task (SMRT)}

The SMRT was used to assess spectral resolution. The SMRT test was chosen over other spectral ripple tasks, because it avoided potential confounds such as loudness and edge-frequency cues whilst still being sensitive to spectral resolution (Aronoff and Landsberger 2013).

Stimuli were $500 \mathrm{~ms}$ long with $100 \mathrm{~ms}$ ramps, nonharmonic tone complexes with 202 equal amplitude pure-tone frequency components, spaced every 1/33.3 octave from 100 to $6400 \mathrm{~Hz}$. The amplitudes of the pure tones were modulated by a sine wave with a $33 \%$ modulation depth. A three-interval, two-alternative forced choice task was used, with a reference stimulus of 20 ripples per octave (RPO) presented at $65 \mathrm{dBA}$. The target stimulus initially had $0.5 \mathrm{RPO}$ and the number of ripples was modified using a 1-up/1-down adaptive procedure with a step size of 0.2 RPO. The test was completed after ten reversals, the last six reversals were averaged to calculate the threshold.

\section{Pitch Ranking Approaches}

The determination of discriminable and indiscriminable electrodes was based on two approaches, acoustic tones presented at the centre frequency of a filter via the System for Testing Auditory Responses (STAR; Saleh et al. 2013) and direct stimulation with biphasic pulses delivered using the NIC.

The pitch ranking task was the same for both approaches. A two-interval twoalternative forced choice paradigm was used. A single presentation consisted of two intervals in which the higher tone was randomly assigned to either the first or second interval, and the listener indicated which interval sounded higher in pitch. The 2 tones/pulses were presented sequentially with duration of $1000 \mathrm{~ms}$ and were $500 \mathrm{~ms}$ apart. Five pairs of tones/pulses were initially presented and responses recorded. If the participant got all five correct the pair was considered discriminable. If they scored less than five a further five presentations were given and scored. If the participant got eight out of ten presentations correct the pair passed. This was based on binomial significance at the $p<0.05$ level (Skellam 1948). A run consisted of presentation of all possible electrode pairs presented either five or ten times, the 
duration of the test varied depending on accuracy of response and number of electrodes activated, but was typically 25 min long for the best performers with a full electrode array but up to $50 \mathrm{~min}$ for poorer performers. For the STAR delivery the stimuli were presented at $65 \mathrm{dBA}$ and the NIC presentation was at $85 \%$ of the upper comfort level (C level). Level roving was applied to each presentation and this was $+/-3 \mathrm{~dB}$ for STAR and $+/-3$ clinical units (CU) for NIC.

The responses from the STAR and the NIC test were highly correlated ( $r h o=0.82$, $N=13, p=0.001$ ) so the results from both tests were combined to create a composite score for each electrode by adding up the passes and fails across both tests thus increasing the calculation power. After determination of the "indiscriminable" electrode set, a "discriminable" electrode set of the same size was selected, using electrodes that were as near as possible to the indiscriminable set and had not failed on either task.

\section{Fitting Procedure for De-activation}

Each electrode in the entire $\mathrm{A} / \mathrm{B}$ set was deactivated. The overall level of the experimental map was checked and if necessary all $\mathrm{C}$ levels adjusted to be approximately equally loud to the optimised clinical map. All other fitting parameters remained at the default settings (900 pulses-per-second, $25 \mu$ s pulse width, eight maxima and MP1+2 stimulation mode).

\section{Results}

Non-parametric statistics (Wilcoxon) were used due to the small number of participants $(N=13)$ and the CCRM and SMRT data exceeded acceptable limits for skewness and kurtosis. Each mapping condition was tested on two occasions and the highest score was used in the analysis.

Fig. 1 shows group results, comparing the optimised clinical map to the "indiscriminable" and "discriminable" deactivation conditions.

For CAPT, CCRM in speech-shaped noise and babble there were no significant differences between any of the mapping options at the $p<0.05$ level. However, the SMRT was significantly poorer for the indiscriminable electrodes deactivated than the clinical map $(z=-2.9, p=0.004)$ and also significantly poorer when the discriminable electrodes were de-activated $(z=-2.62, p=0.009)$. There was no significant difference between the de-activation of the indiscriminable or discriminable electrodes.

\section{Discussion}

Results indicated that there was no measurable benefit for deactivating channels based on pitch ranking for ACE users. Effects were negligible regardless of whether the deactivated channels were indiscriminable or discriminable based on pitch ranking. Care was taken to balance the design and ensure that all maps were used for sufficient time to obtain benefit. Prior to experimental map adjustments, clinical maps were optimised and each participant given an acclimatisation period prior to starting the study. 

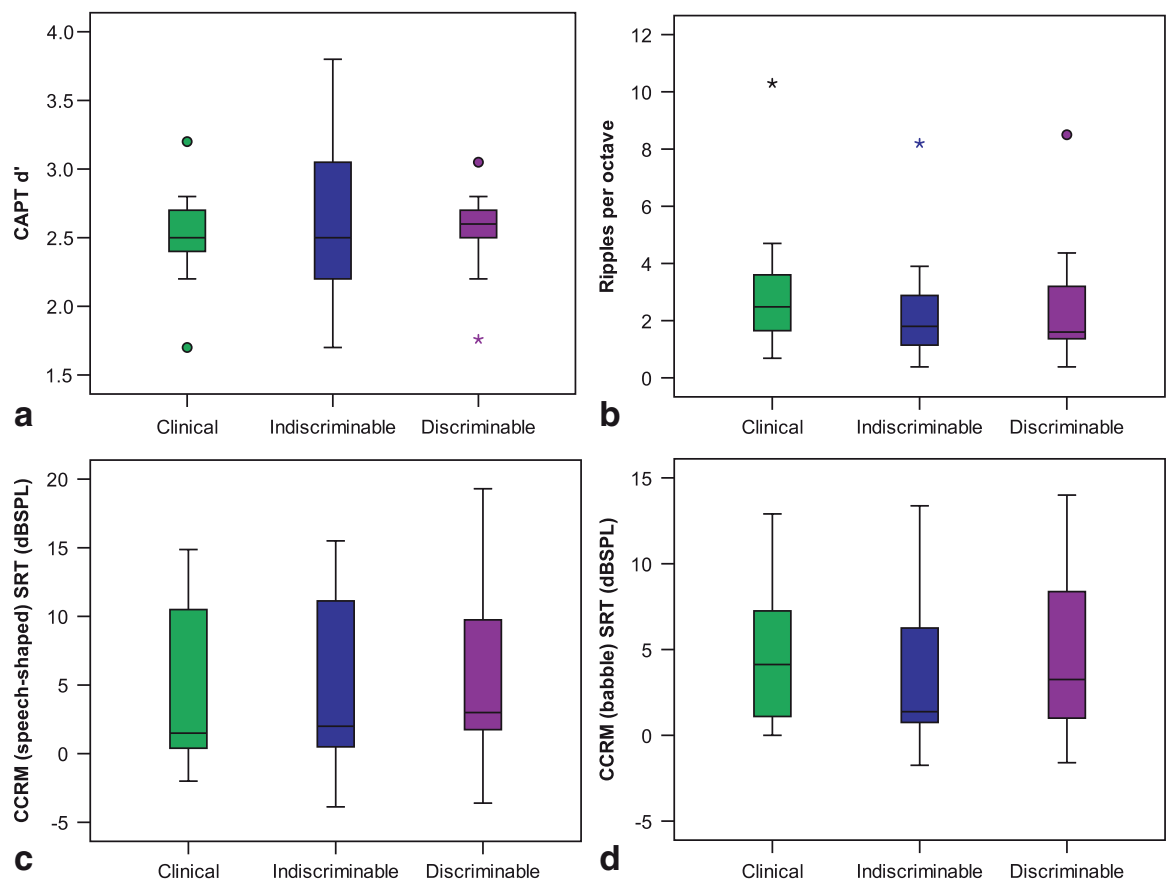

Fig. 1 Boxplots for group results for CAPT d' (panel a), SMRT ripples per octave (panel b), CCRM in speech-shaped noise (panel c), CCRM in babble (panel d). Boxes represent inter-quartile range, the line in the box shows the median and the whiskers indicate range and asterisks and circles show outliers

There was a range in the number of channels selected for deactivation (1-6; median = four channels), which, when combined with the electrodes already switched off, resulted in a de-activation range of 1-10 (median $=$ six channels). For an n-of-m strategy this number of deactivated electrodes may have been too small to demonstrate differences in performance; the selection of channels to deactivate was based purely on electrodes that were indiscriminable from one another in pitch ranking and for many users the number of indiscriminable electrode pairs was low so only small numbers were deactivated.

The only statistically significant finding indicated that spectral resolution was poorer when electrodes were de-activated. When the channels were de-activated the rate of stimulation never changed, however the default filter allocation was used, which may have affected the spectral information (Henshall and McKay 2001). For the deactivation of discriminable channels, the selected channels were as near in site to the deactivated indiscriminable channels in an attempt to avoid dramatic differences between the two conditions for filter allocations. However, for many people the changes to the frequency-to-electrode allocation would have been large when compared to the clinical map.

There should be further exploration of the fitting parameter space for users of n-of-m strategies. The limits or extent of effective use for channel de-activation should be determined and predictive factors to aid the fitting process defined. It is 
also essential to determine the most critical factors to modify for those with poorer performance levels.

Acknowledgments This project was part-funded by Cochlear Research and Development Ltd. Thanks to Stuart Rosen for assistance with speech-in-noise testing.

Open Access This chapter is distributed under the terms of the Creative Commons AttributionNoncommercial 2.5 License (http://creativecommons.org/licenses/by-nc/2.5/) which permits any noncommercial use, distribution, and reproduction in any medium, provided the original author(s) and source are credited.

The images or other third party material in this chapter are included in the work's Creative Commons license, unless indicated otherwise in the credit line; if such material is not included in the work's Creative Commons license and the respective action is not permitted by statutory regulation, users will need to obtain permission from the license holder to duplicate, adapt or reproduce the material.

\section{References}

Aronoff JM, Landsberger DM (2013) The development of a modified spectral ripple test. J Acoust Soc Am 134(2):EL217-EL222

Bolia RS, Nelson WT, Ericson MA, Simpson BD (2000) A speech corpus for multitalker communications research. J Acoust Soc Am 107(2):1065-1066

Brungart DS (2001) Informational and energetic masking effects in the perception of two simultaneous talkers. J Acoust Soc Am 109(3):1101-1109

Friesen LM, Shannon RV, Slattery WH (1999) The effect of frequency allocation on phoneme recognition with the Nucleus-22 cochlear implant. Am J Otol 20:729-734

Garadat SN, Zwolan TA, Pfingst BE (2013) Using temporal modulation sensitivity to select stimulation sites for processor MAPs in cochlear implant listeners. Audiol Neurootol 18(4):247-260

Henshall KR, McKay CM (2001) Optimizing electrode and filter selection in cochlear implant speech processor maps. J Am Acad Audiol 12(9):478-489

Marriage JE, Vickers DA, Baer T, Moore BCJ (2011). Using speech perception measures to guide the choice of amplification. In Seewald RC, Bamford JM (eds) A sound foundation through early amplification. Phonak, Staefa, pp 273-279

Noble JH, Gifford RH, Hedley-Williams AJ, Dawant BM, Labadie RF (2014) Clinical evaluation of an image-guided cochlear implant programming strategy. Audiol Neurootol 19(6):400-411

Saleh SM (2013) Fitting cochlear implants based on pitch perception. Doctoral Dissertation. University College London

Saleh SM, Saeed SR, Meerton L, Moore DR, Vickers DA (2013) Clinical use of electrode differentiation to enhance programming of cochlear implants. Cochlear Implants Int 14:16-18

Skellam JG (1948) A probability distribution Derived from the binomial distribution by regarding the probability of success as variable between the sets of trials. J R Stat Soc Ser B Stat Methodol 10(2):257-261

Vickers D, Backus B, Macdonald N, Rostamzadeh N, Marriage J, Mahon M (2013) Using personal response systems to assess speech perception within the classroom: an approach to determine the efficacy of sound field re-enforcement in primary school classrooms. Ear Hearing 34(4):491-502

Zwolan TA, Collins LM, Wakefield GH (1997) Electrode discrimination and speech recognition in postlingually deafened adult cochlear implant subjects. J Acoust Soc Am 102(6):3673-3685 